\title{
Diabetes Mellitus tipo 2: perfil populacional e fatores associados à adesão terapêutica em Unidades Básicas de Saúde em Porto Velho-RO
}

\author{
Diabetes Mellitus tipo 2: population profile and factors associated with therapeutic \\ adherence in Basic Health Units in Porto Velho-RO
}
Diabetes Mellitus tipo 2: perfil de la población y factores asociados con la adherencia terapéutica en las Unidades Básicas de Salud en Porto Velho-RO

Adriane Bonotto Salin ${ }^{1 *}$, Monalysa Silva Nogueira Bandeira ${ }^{1}$, Pâmela Regina Nunes de Oliveira Freitas $^{2}$, Idelma Serpa ${ }^{2}$.

\section{RESUMO}

Objetivo: Estabelecer o perfil dos pacientes diabéticos tipo 2 e fatores associados à adesão terapêutica em Unidades Básicas de Saúde nas quatro regiões do município de Porto Velho. Métodos: Optou-se por uma pesquisa quantitativa de caráter descritivo, feita com uso de 2 instrumentos, um formulário com 17 perguntas abordando o perfil sócio demográfico e um questionário com 7 perguntas fechadas, sobre hábitos de vida. Resultados: Após a análise dos dados coletados comprovou-se um predomínio do sexo feminino com $62 \%$ e apenas $38 \%$ masculino, e com $27 \%$ na faixa etária entre 56 até 65 anos, quanto à escolaridade, $30 \%$ possuem o ensino fundamental incompleto, $46 \%$ informaram renda familiar mensal de até um salário mínimo, $61 \%$ informaram estado civil casado/união estável. Identificou-se também, outros fatores de risco de grande relevância da pesquisa, estão implicados para desenvolvimento da Diabetes, que contribuem para o não seguimento medicamentoso, são: dificuldade no seguimento medicamentoso na unidade com $37 \%$, efeitos colaterais com $23 \%$, esquecimento com $21 \%$ e dentre outros com $36 \%$. Conclusão: Acredita-se que a adoção de novos hábitos e estilo de vida através de uma mudança na promoção à educação em saúde com rastreamento e monitoramento dos fatores de risco e que contribua para novos estudos.

Palavras-chave: Diabetes Mellitus tipo 2, Unidade, Fatores de Risco.

\begin{abstract}
Objective: To establish the profile of type 2 diabetic patients and factors associated with therapeutic adherence in Basic Health Units in the four regions of the city of Porto Velho. Methods: We used a quantitative research of a descriptive character, using two instruments, a form with 17 questions addressing the sociodemographic profile and a questionnaire with 7 closed questions about life habits. Results: After analyzing the data collected, a predominance of females was found, with $62 \%$ and only $38 \%$ male, and $27 \%$ between 56 and 65 years of age, in relation to schooling, $30 \%$ had incomplete primary education, $46 \%$ reported monthly family income of up to one minimum wage, $61 \%$ reported married / stable marital status. It was also identified other risk factors of great relevance of the research, are implicated for the development of Diabetes, which contribute to the non-follow-up medication, are: difficulty in following the drug in the unit with $37 \%$, side effects with $23 \%$, oblivion with $21 \%$ and among others with $36 \%$. Conclusion: It is believed that the adoption of new habits and lifestyle, through a shift in promotion to health education, with tracking and monitoring of risk factors and contributing to further studies.
\end{abstract}

Keywords: Type 2 Diabetes Mellitus, Unit, Risk Factors.

\footnotetext{
1Faculdade Interamericana de Porto Velho (UNIRON), Porto Velho/Rondônia. *E-mail: adrybonotto@gmail.com

2 Instituto Universitário Italiano de Rosário - IUNIR - Rosário / Argentina.
} 


\section{RESUMEN}

Objetivo: Establecer el perfil de los pacientes diabéticos tipo 2 y los factores asociados con la adherencia terapéutica en las Unidades Básicas de Salud en las cuatro regiones de la ciudad de Porto Velho. Métodos: utilizamos una investigación cuantitativa de carácter descriptivo, utilizando dos instrumentos, un formulario con 17 preguntas que abordan el perfil sociodemográfico y un cuestionario con 7 preguntas cerradas sobre hábitos de vida. Resultados: Después de analizar los datos recolectados, se encontró un predominio de mujeres, con $62 \%$ y solo $38 \%$ hombres, y $27 \%$ entre 56 y 65 años de edad, en relación con la educación, el $30 \%$ tenía educación primaria incompleta, El 46\% reportó ingresos familiares mensuales de hasta un salario mínimo, el $61 \%$ informó el estado civil conyugal / estable. También fue identificado otros factores de riesgo de gran relevancia de la investigación, están implicados en el desarrollo de la diabetes, que contribuyen a la no-droga de seguimiento son: dificultad en el seguimiento médico en la unidad a un $37 \%$, los efectos secundarios con el $23 \%$, se olvide $21 \%$ y entre otros con un $36 \%$. Conclusión: Se cree que la adopción de nuevos hábitos y estilos de vida, a través de un cambio en la promoción a la educación para la salud, con el seguimiento y monitoreo de los factores de riesgo y la contribución a más estudios.

Palabras clave: Diabetes mellitus tipo 2, Unidad, Factores de riesgo.

\section{INTRODUÇÃO}

Devido ao processo de envelhecimento populacional o aumento e o surgimento de doenças crônicas incapacitantes, passaram a ganhar uma maior evidência no cenário da saúde pública. Entre elas destaca-se a diabetes mellitus (DM), que é uma das doenças crônicas mais comuns entre pessoas obesas e com mais de 40 anos. Esta patologia além de ocasionar maior ônus para os indivíduos, famílias e Estado, compõem uma das principais causas de incapacidades e morbimortalidade (ROEDIGER, MA et al 2018; Silva AL et al. 2017).

Diabetes mellitus é uma doença metabólica crônica não transmissível de origem multifatorial com alta frequência em todo mundo caracterizada pela elevação permanente dos níveis glicêmicos decorrente da ausência ou incapacidade da insulina de exercer sua função, gerando complicações e disfunções de órgãos essenciais. Sua prevalência tem aumentado proporcionalmente com a obesidade resultante de mudanças de estilo de vida na modernidade (MOREIRA CA et al., 2019).

Pertermann XB et al. (2015) cita ainda que existam tipos distintos de diabetes, ocasionados por uma interação de fatores genéticos, ambientais e de estilo de vida, no entanto salienta que são os fatores ambientais que afetam de forma direta na adesão desta patologia.

Para Zimmet P e Alberti GK (2016), o Brasil já ocupa a quarta posição entre os países com maior número de pessoas vivendo com DM, aproximadamente 14,3 milhões, atrás apenas da China, Índia e dos Estados Unidos.

A Organização Mundial da Saúde (OMS) prevê que, em 2030, o Brasil tenha uma população de aproximadamente 11,3 milhões de diabéticos, dos quais aproximadamente $33 \%$ terão entre 60 e 79 anos (BRASIL, 2014).

O DM tipo 2 está relacionado a 90\% - 95\% dos casos, e a causa da ocorrência está relacionada a resistência da ação da insulina e a resposta secretora inadequada caracteriza-se pela deficiência da insulina, manifesta-se pela incapacidade de compensar essa resistência (PETERMANN XB et al., 2015).

O desenvolvimento do diabetes mellitus tipo 2 (DM2) pode ser determinado por fatores genéticos e ambientais, o sobrepeso, a obesidade, a alimentação desequilibrada e sedentarismo aumentam a possibilidade de desenvolver DM e como nem todos os pacientes conseguem alcançar os objetivos do tratamento, eles frequentemente desenvolvem estas complicações que irão afetar sua qualidade de vida e consequentemente aumentam o custo dos seus cuidados e tratamento (SOUZA MR et al., 2015). 
Nos casos de diabetes mellitus tipo 2 ocorrem desordens metabólica de etiologia múltipla, apresentando distúrbios no metabolismo de carboidratos, proteínas e lipídeos causados pela resistência tecidual periférica ou ausência de resposta insulínica associada a uma deficiência relativa da insulina, os quais cooperam para o desenvolvimento da hiperglicemia (BRUNO A et al., 2014).

O DM depois de instalado, necessita que o paciente adira ao tratamento, que inclui alimentação saudável, prática de exercícios físicos, monitorização da glicemia, manutenção da integridade e função dos pés, uso de medicamentos e cessação do tabagismo e fundamental para a prevenção das complicações (SOUZA JD et al., 2017).

No Brasil, o Ministério da Saúde, criou vários programas de controles de doenças de maior impacto na população, e no caso do diabetes mellitus, foi criado em 2002 o Programa Nacional de Hipertensão e Diabetes Mellitus - Hiperdia, na tentativa de reorientar a assistência farmacêutica para o fornecimento contínuo do medicamento, assim como monitoramento das condições clínicas dos usuários dos serviços de saúde (CORTEZ DN et al., 2015).

A adesão ao tratamento, ou seja, seguir as recomendações preconizadas por um profissional de saúde como: tomar medicamentos, seguir o plano alimentar ou adotar mudanças no estilo de vida é essencial para prevenção ou retardo das complicações agudas e crônica do DM, bem como promoção da qualidade de vida, redução da mortalidade. No entanto os maiores problemas que profissionais da saúde se deparam durante a intervenção com pacientes portadores de DM em tratamento é a baixa adesão terapêutica, uma vez que requerem a participação e a colaboração dos pacientes para mudanças nos hábitos de vida. Frente a isso o estudo teve como objetivo estabelecer o perfil dos pacientes diabéticos tipo 2 e fatores associados adesão terapêutica em Unidades Básicas de Saúde em Porto Velho-Rondônia.

\section{MÉTODOS}

Estudo de campo de enfoque prospectivo, exploratório de abordagem quantitativa. A pesquisa foi realizada Unidades Básicas de Saúde (UBS), nas quatro regiões do município de Porto Velho no Estado de Rondônia, Brasil. A amostra compreendeu de 205 portadores de diabetes mellitus tipo 2. Foram incluídos na pesquisa os portadores de diabetes tipo 2, maiores de 18 anos que estavam nas unidades aguardando consulta médica e nos grupos do Hiperdia, no dia da coleta de dados e que aceitaram voluntariamente participar da pesquisa e assinando o TCLE. Foram excluídos da pesquisa portadores com distúrbios neurológicos e mentais comprovados e os portadores de diabetes mellitus tipo 1 e os pacientes que não assinaram o TCLE ou se recusaram a participar da pesquisa. O estudo foi desenvolvido obedecendo ao estabelecido na Resolução n. 466/2012/CNS/MS.

Em virtude de se tratar de uma pesquisa com seres humanos, o estudo foi submetido ao Comitê de Ética em Pesquisa (CEP) e após parecer favorável, iniciou-se a coleta de dados. A pesquisa dividiu-se através de duas etapas: a primeira foi constituída na consulta de informações adquiridas mediante um apanhado bibliográfico, para constituir o embasamento teórico, e a segunda foi a pesquisa de campo, cujo trabalho se desenvolveu a aplicação de dois instrumentos, um formulário com 17 perguntas objetivas, abordando características sóciodemográficas, econômicas e de saúde da população bem como fatores que afetam a adesão no tratamento ao portador diabético tipo 2, e outro um questionário com 7 perguntas fechadas, lembrando que o questionário validado de atividades com o diabetes (QAD), este buscou averiguar os cuidados do paciente diabético a adesão ao tratamento farmacológico e não farmacológico, em busca de um levantamento palpável que existe um número grande de portadores de diabetes mellitus tipo 2 .

A coleta de dados foi a partir da Resolução n. ${ }^{510}$, de 7 abril de 2016, do Conselho Nacional de Saúde. Cabe ressaltar que não houve interferência das pesquisadoras durante as respostas. O presente estudo é resultado parcial de um estudo que foi submetido à apreciação do CEP da Faculdades Integradas Aparício Carvalho Ltda. - FIMCA, a qual expediu parecer favorável sob o n.ํ․․ 2.618 .527 e assinatura do termo de Consentimento Livre e Esclarecido (TCLE). 


\section{RESULTADOS}

Os dados foram obtidos a partir de 205 pacientes portadores de Diabetes Mellitus tipo 2 cadastrados nas Unidades Básicas de Saúde, no município de Porto Velho/RO. Além disso, os resultados possibilitaram realizar o perfil e o autocuidado dos portadores de diabetes tipo 2.

No primeiro período visou traçar o perfil dos entrevistados, com as informações obtidas do gênero: idade, estado civil, cor/raça, grau de escolaridade, renda familiar; seguida dos dados sobre práticas de hábitos e estilo de vida, como: etilismo, tabagismo, atividade física, controle alimentar, antecedentes de diabetes na família e uso de medicações para diabetes. E fatores que contribuem o não seguimento medicamentoso, como: dificuldade para conseguir o medicamento na unidade, falta de orientação quanto a forma de tomar a medicação, efeitos colaterais, prefere remédios caseiros, esquecimento e acha que o diabetes está controlado, pois não apresenta sinais e sintomas e as dificuldades enfrentadas pelos pacientes. Dentre os fatores de grande relevância para a nossa pesquisa estão as atividades de autocuidado com o diabetes tipo 2, durante 7 dias das semanas, práticas para 0 autocuidado, como: seguir a dieta saudável e alimentação especifica frutas/vegetais, monitorização da glicemia, realizam o autoexame dos pés.

Tabela 1- Perfil sócio demográfico dos portadores de DM tipo 2, nas UBS das quatro regiões. Porto Velho Rondônia, 2019 ( $n=205)$.

\begin{tabular}{|c|c|c|c|}
\hline & Variável & $\mathbf{N}$ & $\%$ \\
\hline \multicolumn{4}{|l|}{ Sexo } \\
\hline & Masculino & 71 & $38 \%$ \\
\hline \multicolumn{4}{|l|}{$\begin{array}{l}\text { Feminino } \\
\text { Idade }\end{array}$} \\
\hline & 18 a 25 & 9 & $5 \%$ \\
\hline & 26 a 35 & 15 & $7 \%$ \\
\hline & 36 a 45 & 23 & $11 \%$ \\
\hline & 46 a 55 & 35 & $17 \%$ \\
\hline & 56 a 65 & 56 & $27 \%$ \\
\hline & 66 a 75 & 45 & $22 \%$ \\
\hline & $>76$ & 22 & $11 \%$ \\
\hline \multicolumn{4}{|c|}{ Grau de escolaridade } \\
\hline & Analfabeto & 25 & $12 \%$ \\
\hline & Ensino fundamental completo & 37 & $18 \%$ \\
\hline & Ensino fundamental incompleto & 62 & $30 \%$ \\
\hline & Ensino médio completo & 24 & $12 \%$ \\
\hline & Ensino médio incompleto & 31 & $15 \%$ \\
\hline & Ensino superior completo & 15 & $7 \%$ \\
\hline & Ensino superior incompleto & 11 & $6 \%$ \\
\hline \multicolumn{4}{|c|}{ Renda familiar } \\
\hline & Não tem & 22 & $11 \%$ \\
\hline & Menos de um salário & 30 & $15 \%$ \\
\hline & 1 salário & 94 & $46 \%$ \\
\hline & 2 salários & 53 & $26 \%$ \\
\hline & 3 a 4 salários & 6 & $3 \%$ \\
\hline \multicolumn{4}{|c|}{ Estado civil } \\
\hline & Casado / união estável & 115 & $61 \%$ \\
\hline & Solteiro (a) & 36 & $18 \%$ \\
\hline & Separado (a) & 17 & $8 \%$ \\
\hline & Viúvo (a) & 27 & $13 \%$ \\
\hline \multicolumn{4}{|l|}{ Raça } \\
\hline & Branco & 36 & $18 \%$ \\
\hline & Negro & 41 & $20 \%$ \\
\hline & Amarelo & 17 & $8 \%$ \\
\hline & Pardo & 98 & $48 \%$ \\
\hline & Indígena & 13 & $6 \%$ \\
\hline
\end{tabular}

Fonte: Dados das pesquisadoras (2019). 
Na tabela 1, a distribuição do perfil sociodemográfico evidenciou-se predominância do gênero feminino $62 \%$. Ademais, destaca-se no quesito faixa etária foi prevalente (27\%) entre 56 a 65 anos, $(30 \%)$ tem o ensino fundamental incompleto, (46\%) possuem renda familiar mensal de até 1 salário mínimo, $(61 \%)$ informaram estado civil casado/união estável e (48\%) declararam-se pardos, conforme Tabela 1.

Tabela 2 - Hábitos e estilo de vida na visão geral, Porto Velho - Rondônia, 2019 ( $n=205)$.

\begin{tabular}{|c|c|c|}
\hline Classificação & $\mathbf{N}$ & $\%$ \\
\hline \multicolumn{3}{|l|}{ Etilismo } \\
\hline Sim & 19 & $9 \%$ \\
\hline Não & 134 & $65 \%$ \\
\hline Bebeu anteriormente & 52 & $26 \%$ \\
\hline \multicolumn{3}{|l|}{ Tabagismo } \\
\hline Sim & 9 & $4 \%$ \\
\hline Não & 155 & $76 \%$ \\
\hline Fumou anteriormente & 41 & $20 \%$ \\
\hline \multicolumn{3}{|l|}{ Atividade física } \\
\hline Sim & 39 & $22 \%$ \\
\hline Não & 99 & $55 \%$ \\
\hline Praticava anteriormente? & 67 & $23 \%$ \\
\hline \multicolumn{3}{|l|}{ Controle alimentar } \\
\hline 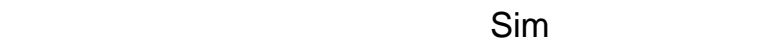 & 168 & $89 \%$ \\
\hline Não & 37 & $11 \%$ \\
\hline \multicolumn{3}{|l|}{ Antecedentes de diabetes na família } \\
\hline Sim & 174 & $82 \%$ \\
\hline Quem? & Mãe / 81 & $32 \%$ \\
\hline Não & 31 & $18 \%$ \\
\hline \multicolumn{3}{|l|}{ Uso de medicações para diabetes } \\
\hline Sim & 197 & $96 \%$ \\
\hline Não & 8 & $4 \%$ \\
\hline Antidiabéticos orais & 112 & $57 \%$ \\
\hline Insulina & 53 & $27 \%$ \\
\hline Antidiabéticos orais e insulina & 32 & $16 \%$ \\
\hline
\end{tabular}

Fonte: Dados das pesquisadoras (2019).

A tabela 2, destaca-se os hábitos e estilo de vida na visão geral, de maior relevância no que diz respeito à atividade física (55\%) dos participantes declaram não praticar nenhuma atividade física, já a alimentação (89\%) confirmou fazer o controle alimentar, nos casos de antecedentes de diabetes na família (82\%) possuem algum familiar portador de Diabetes Mellitus tipo 2, relacionado aos que afirmaram possuir algum membro da família a prevalência da mãe foi de (32\%), quanto ao uso das medicações para tratamento do Diabetes Mellitus tipo 2, (96\%) afirmam fazer uso de algum tipo de medicamento, dentre esta porcentagem como maioria (57\%) fazem uso de antidiabéticos orais e $(27 \%)$ apenas de insulina, dos participantes que fazem uso tanto das medicações quanto das insulinas representam (16\%).

Tabela 3 - Fatores que contribuem para o não seguimento medicamentoso. Porto Velho - Rondônia, 2019 $(n=205)$.

\begin{tabular}{|c|c|c|}
\hline Variável & $\mathbf{N}$ & $\%$ \\
\hline Dificuldade para conseguir o medicamento na unidade & 72 & $37 \%$ \\
\hline Efeitos colaterais & 46 & $23 \%$ \\
\hline Esquecimento & 54 & $21 \%$ \\
\hline Outros & 25 & $36 \%$ \\
\hline
\end{tabular}

Fonte: Dados das pesquisadoras (2019). 
Afirmam na tabela 3, destaca-se relevância na pesquisa, existem fatores que contribuem para o não seguimento medicamentoso, dentre eles destaca-se os principais, enquanto a dificuldades para conseguir medicamentos nas unidades $(37 \%)$ dos participantes, já $(23 \%)$ relaram sentir efeitos colaterais, enquanto $(21 \%)$ dizem esquecer-se de tomar as medicações.

Tabela 4 - $O$ auto cuidado dos pacientes portadores de diabetes tipo 2. Porto Velho - Rondônia, 2019 ( $n=$ 205).

\begin{tabular}{ccc}
\hline Variável & $\mathbf{N}$ & $\mathbf{\%}$ \\
\hline Segue dieta saudável & 37 & $18 \%$ \\
Não pratica & 93 & $45 \%$ \\
1 a 2 dias por semana & 40 & $20 \%$ \\
3 a 4 dias por semana & 23 & $11 \%$ \\
5 a 6 dias por semana & 12 & $6 \%$ \\
Todos os dias & & \\
Nlimentação especifica frutas/vegetais & 37 & $18 \%$ \\
Não consome & 101 & $49 \%$ \\
1 a 2 dias na semana & 36 & $18 \%$ \\
3 a 4 dias na semana & 19 & $9 \%$ \\
5 a 6 dias na semana & 12 & $6 \%$ \\
Todos os dias & & \\
Monitorização da glicemia & 0 & $0 \%$ \\
Não verificam & 119 & $58 \%$ \\
1 a 2 dias por semana & 45 & $22 \%$ \\
3 a 4 dias por semana & 26 & $13 \%$ \\
5 a 6 dias por semana & 15 & $7 \%$ \\
Todos os dias & & \\
\hline Realizam o auto exame dos pés & 72 & $35 \%$ \\
Nunca & 33 & $16 \%$ \\
1 a 2 dias na semana & 41 & $20 \%$ \\
3 a 4 dias na semana & 32 & $16 \%$ \\
5 a 6 dias na semana & 27 & $13 \%$ \\
Todos os dias & &
\end{tabular}

Fonte: Dados das pesquisadoras (2019).

A tabela 4, apresenta os dados referente ao autocuidado dos pacientes portadores de diabetes tipo 2, com sua maioria de (45\%) praticam de 1 a 2 vezes na semana uma alimentação correta, os (49\%) relatam comer de 1 a 2 vezes por sema frutas e legumes. Nos grupos do hiperdia todos os pacientes realizam o teste de glicemia capilar, sendo assim (58\%) realizam a glicemia capilar de 1 a 2 vezes por semana.

Referente ao autoexame dos pés (35\%) relataram nunca ter realizado o exame, (16\%) afirmam realizam de 1 a 2 vezes por semana o exame, já (20\%) dizem fazer o autoexame de 3 a 4 vezes na semana, dos entrevistados (16\%) diz fazer o exame de 5 a 6 vezes por semana e apenas (13\%) fazem o autoexame todos os dias.

\section{DISCUSSÃO}

A população amostral foi de 205 participantes, encontrados nos atendimentos médicos e dos grupos Hiperdia que participaram da pesquisa.

Os dados foram analisados de acordo com os objetivos propostos, sendo agrupados em gráficos e tabelas para o melhor entendimento e classificados nas seguintes categorias: através de dois métodos sendo um formulário, abordando características sócio demográficas e um questionário de atividades com o diabetes 
(QAD), este buscou averiguar os cuidados do paciente diabético a adesão ao tratamento farmacológico e não farmacológico.

Por meio desse estudo observou-se um predomínio do sexo feminino com $62 \%$ do total da amostra, fato este pode estar relacionado a cultura brasileira, a procura das mulheres por atendimento de prevenção e predominante no que diz respeito à saúde.

Levorato CD et al. (2014) discorre que público masculino como o de ser o provedor, relacionado ao trabalho e à família, dominador, a quem compete o poder nas relações de gênero, deixando em segundo plano cuidados básicos.

Costa-Junior FM et al. (2016) acrescenta ainda que a masculinidade mostrar como ideal a imagem de homens ativos e fortes, e neste padrão o grupo é visto como não vulneráveis, fato que reduz a procura masculina por serviços preventivos, aumenta a mortalidade e impedindo que estes sintam acolhidos nos serviços de saúde.

Em relação à escolaridade $30 \%$ tem o ensino fundamental incompleto, o grau de escolaridade é um fator facilitador de risco para o desenvolvimento de doenças ou condições crônicas, o que pode ser um problema a ser considerado pela equipe e gestores atuantes na unidade de saúde da família, pelo número preocupante de pessoas com baixa escolaridade, ou seja, pessoas que podem estar mais predispostas a adoecer (SATO TO et al., 2017).

Quanto a renda familiar a maioria dos pacientes $46 \%$ afirmam renda familiar mensal de até 1 salário mínimo, esse fator pode interferir negativamente para a não adesão a hábitos saudáveis interferindo diretamente na qualidade de vida destes pacientes, o baixo rendimento relacionado aos preços dos alimentos atuam como uma barreira para que as famílias de baixa renda façam escolhas alimentares adequadas (BORGES CA et al., 2015).

Neste estudo foram referidos que $61 \%$ dos participantes são casados ou vive em união estável, a presença de um parceiro é condição importante no manejo da doença por parte de indivíduos diabéticos, uma vez que buscam incentivar os pacientes a aderirem ao tratamento, para controlar hábitos não saudáveis e adotar estilos de vida favoráveis. Caracterizou os usuários portadores de diabetes tipo 2 da atenção primária à Saúde segundo aspectos demográficos, socioeconômicos, hábitos e estilo de vida foram constatados que em sua maioria $(64,6 \%)$ dos usuários era casado (a) ou com união estável e não variou entre as regiões, porém as proporções de união estável no Norte e Nordeste foram maiores do que nas demais. Na variável cor auto referidas, a maior proporção foi de pardos $(50,5 \%)$, porém maior no Norte, Nordeste e Centro-Oeste (GUIBU IA et al., 2017).

O etilismo em um âmbito geral foi de (9\%) para os que fazem uso do álcool, (26\%) fizeram uso anteriormente, mas abandonaram a prática e $65 \%$ nunca fizeram ingestão de bebida alcoólica. Por ser considerada uma droga lícita o seu uso é considerável, desde que sua frequência não seja exacerbada, pois assim tornam-se fatores de risco não apenas para diabetes, mas para outras doenças e agravos não transmissíveis diversas como cirrose, câncer, acidentes dentre outros (VITOR IO et al., 2014).

Apenas nos achados de Silva AL et al. (2017), realizado na cidade de Viçosa-MG, que teve como objetivo analisar o nível de atividade física, o estado nutricional autorreferido, foi observada frequência elevada de inatividade física, excesso de peso, sendo que os homens apresentaram uma frequência superior quando comparados às mulheres. Neste estudo $55 \%$ dos participantes não praticam nenhum tipo de atividade física é um número alto a se comparar com os benefícios da atividade física para boa manutenção da qualidade de vida. Dos participantes (89\%) dizem fazer controle alimentar, o que é favorável para o controle do Diabetes. O Guia Alimentar para a População Brasileira destaca o quanto e importante o consumo regular de legumes e verduras, pois além de muito saudáveis possuem potentes valores nutritivos (BRASIL, 2014).

Um dos fatores importantes em algumas doenças incluindo o Diabetes Mellitus é o histórico familiar, se confirmado neste estudo onde $(82 \%)$ relataram ter algum membro da família portador do Diabetes Mellitus Tipo 2. O diabetes é uma doença crônica sistêmica requer medicamentos eficazes e de segurança adequado 
ao uso contínuo. O esquema terapêutico de primeira escolha para o tratamento do diabetes tipo 2 geralmente envolve uma combinação de agentes hipoglicemiantes orais de classes terapêuticas diferentes (ALVES da CR et al., 2017).

Uma parcela da população da pesquisa, (37\%), justifica a não adesão a terapêutica é pela dificuldade para adquirir os medicamentos, buscar um autor que fala dessa falta de medicamentos no SUS, implicam segundo a pesquisa pela baixa disponibilidade de medicamentos no setor público é um problema global. Os medicamentos são de grande importância no tratamento de morbidades que apresentam prevalências crescentes no Brasil devido à perspectiva da longevidade, exemplo das doenças crônico-degenerativas (hipertensão arterial sistêmica e diabetes mellitus), e problemas de saúde mental (NASCIMENTO RCRM, et al., 2017).

Nos deparamos com um número de diabéticos que mencionam não seguir o tratamento, meramente por esquecimento, ou seja, (21\%) dos pacientes esquecem de fazer uso das medicações. Tal fato pode ser justificado pelo fator da idade será desta pesquisa que foi de 56 a 65 anos, o esquecimento e o uso dos medicamentos foram causas para não adesão total ao tratamento. Caracterizam-se comportamentos involuntários e não intencionais e assemelham se aos problemas relacionados com outras patologias relacionadas a idade, podem ser melhorados por estratégias de educação e orientação da equipe, quanto à participação da família (STEFANO ICA et al., 2017).

Já na alimentação específica (45\%) realiza uma dieta saudável de 1 a 2 vezes por semana, e um número baixo segundo o recomendado pela Organização Mundial de Saúde é de 400 gramas diários, em cinco ou mais dias da semana. Tornando-se o ideal aderir ao consumo diário três porções de frutas e duas de verduras e legumes (ASBRAN, 2015).

Segundo o Guia Alimentar para a População Brasileira é importante o consumo regular de legumes e verduras. Além de serem muito saudáveis são excelentes fontes de vitaminas e minerais e, portanto, muito importantes para a prevenção de deficiências de micronutrientes, fornecem, de um modo geral, muitos nutrientes e possui baixo teor calórico, tornando-se ideais para a prevenção da obesidade e das doenças crônicas associadas a esta condição, como o diabetes e doenças do coração (BRASIL, 2014).

Os testes de glicemia refletem o nível glicêmico atual e instantâneo no momento exato do teste, na monitorizarão da glicemia capilar realizada pelos pacientes do grupo hiperdia foi de (58\%) realizam de 1 a 2 vezes por semana. Foram de grande relevância os dados obtidos nesta pesquisa que (35\%) dos pacientes que nunca realizaram o autoexame dos pés, e não sabiam a importância do autoexame, e como é um simples procedimento e pode ser realizado pelo próprio paciente em casa.

Este fator pode ser explicado devido à baixa escolaridade, sendo apontada como fator de risco importante em alguns estudos os quais indicam que o baixo grau de instrução é um forte aliado ao desenvolvimento de complicações, por influenciar a capacidade dos indivíduos de assimilarem os conhecimentos sobre a doença e a importância dada ao controle do DM, incluindo aí os cuidados preventivos às complicações com os pés (BOELL JEW, et al., 2014).

\section{CONCLUSÃO}

Este estudo é de grande relevância para a sociedade em que vivemos, os resultados apresentam um diagnóstico parcial do estilo de vida dos usuários na Atenção primária, a maioria dos pacientes possuíam antecedentes familiares de DM, apontou um predomínio da doença entre usuários de produção e idosos., que não tem habito de praticar atividade física e realizam o autocuidado e o tratamento, no entanto nem sempre da forma adequada. Estudo aponta um dado preocupante a dos participantes do estudo não tem o autoconhecimento para avaliar seus pés, somente percebendo a gravidade do estágio da doença quando tem um membro amputado. Acredita-se que este estudo vai colaborar para busca de práticas e aprimoramento d estratégias para adesão ao tratamento e autocuidado a fim evitar comorbidades relacionadas a DM beneficiando desta forma a promoção, prevenção e recuperação da saúde aos portadores da DM tipo 2 . Bem como fomentar novos estudos e pesquisas sobre o tema. 


\section{REFERÊNCIAS}

1. ALVES C, et al. Fármacos para o Tratamento do Diabetes Tipo II: Uma Visita ao Passado e um Olhar para o Futuro, Rev. Virtual Quim. 2017, 9 (2), 514-534.

1. ASBRAN, Associação Brasileira de Nutrição. Notícias: Ainda é baixo o consumo de verduras e frutas pelo brasileiro. Publicado em 09/04/2015.

2. BOELL JEW, et al. Fatores de risco para o desencadeamento do pé diabético. Revista Eletrônica de Enfermagem. 2014 abr/jun;16(2):386-93.

3. BORGES CA, et al. Quanto custa para as famílias de baixa renda obterem uma dieta saudável no Brasil?. Caderno. Saúde Pública, Rio de Janeiro. Jan, 2015. 31(1):137-148.

4. BRASIL. Portal Brasil. Saúde do idoso. Brasília. 2014 [citado em 2014 ago 21].

5. BRASIL. Ministério da Saúde. Guia alimentar para a população brasileira. Brasilia-DF. 2014.

6. BRUNO, Arelli; PEREIRA, Luciene Rabelo; ALMEIDA, Herivelto dos Santos. Avaliação da prevalência de fatores de risco para o desenvolvimento de diabetes mellitus tipo 2 em pacientes da Clínica Unesc Saúde. Demetra: alimentação, nutrição \& saúde. Demetra; 2014; 9(3); 661-680.

7. CORTEZ, Daniel Nogueira et al. Complicações e o tempo de diagnóstico do diabetes mellitus na atenção primária. Acta Paul Enferm. 2015; 28(3):250-5.

8. COSTA, Junior Florencio Mariano et al. Gênero e cuidados em saúde: Concepções de profissionais que atuam no contexto ambulatorial e hospitalar. Sexualidad, Salud y Sociedad - Revista Latinoamericana ISSN 1984-6487 / n. 23 - ago. / ago. / aug. 2016 - pp.97-117.

9. STEFANO, Isabel Cristina Aparecida et al. Uso de medicamentos por idosos: análise da prescrição, dispensação e utilização num município de porte médio do estado de São Paulo. Rev.Bras. Geriatr. Gerontol. Rio de Janeiro, 2017; 20(5): 681-692.

10. GUIBU, Ione Aquemi et al. Características principais dos usuários dos serviços de atenção primária à saúde no Brasil. Revista Saúde Pública. 2017. https://doi.org/10.11606/S1518-8787.2017051007070.

11. LEVORATO, Cleice Daiana et al. Fatores associados à procura por serviços de saúde numa perspectiva relacional de gênero. Ciência \& Saúde Coletiva, Ribeirão Preto-SP, 2013.

12. 19(4): 1263-1274.

13. MOREIRA, Carolina A et al. Novos conceitos em diabetes e metabolismo ósseo. J. Bras. Nefrol. São Paulo, Dec. 2015, Available from. v. 37, n. 4, p. 490-495.

14. NASCIENTO, Renata Cristina Rezende Macedo et al.Disponibilidade de medicamentos no SUS. Revista de saúde pública. 2017;51 Supl 2:10s. https://doi.org/10.11606/S1518-8787.2017051007062.

15. ROEDIGER, Manuela de Almeida et al. Diabetes mellitus referida: incidência e determinantes, em coorte de idosos do município de São Paulo. Ciência \& Saúde Coletiva. Rio de Janeiro, v. 23, n. 11, p. 3913-3922, nov. 2018.

16. SATO, Tatiana de oliveira et al. Doenças Crônicas Não Transmissíveis em Usuários de Unidades de Saúde da Família - Prevalência, Perfil Demográfico, Utilização de Serviços de Saúde e Necessidades Clínicas. Rev. Bras. de Ciências da Saúde. 2017. DOI:10.4034/RBCS.2017.21.01.05. Volume 21 Número 1 Páginas 35-42 2017 ISSN 1415-2177.

17. SILVA, Raquel Rodrigues de Almeida; FERREIA, Fabricia Geralda; SAGHETO; Wellington. Atividade física no lazer, estado nutricional autoreferido e tempo gasto sentado em trabalhadores do comércio. Revista Brasileira de Nutrição Esportiva, São Paulo. v. 10. n. 56. p.222-229. Mar./Abr. 2016. ISSN 1981-9927.

18. SOUZA, Jackline Duran et al. Adherence to diabetes mellitus care at three levels of health care. Esc. Anna Nery, Rio de Janeiro. 2017, v. 21, n. 4, e20170045.

19. SOUSA, MR, MCINTYRE T, MARTINS T, et al. Questionário dos Conhecimentos da Diabetes (QCD): propriedades psicométricas. Rev. Port. Sau. Pub., Lisboa, v. 33, n. 1, p. 33-41, jun. 2015. DOI.org/10.1016/j.rpsp.2014.07.002.

20. PETERMANN, Xavele Braatz et al. Epidemiologia e cuidado à Diabetes Mellitus Mostra Interdisciplinar do curso de Enfermagem, Volume 2, Número 01, Jun. 2015 Praticado na Atenção Primária à Saúde: uma revisão narrativa. Saúde (Santa Maria) 2015, v.41,n.1,p.49-56.

21. VITOR, lara de Oliveira; BEVIDELLI, Maia Meimel; COUTINHO, Raquel Machado Cavalca. Prevalência de fatores de risco para doenças crônicas não transmissíveis entre graduandos de enfermagem: diferença de gêneros. $J$ Health Sci Inst. 2014; Vol. 32 n. (4):390-5.

22. SCULL, Juan Alberto Abreu. Prevenção De Complicações Em Adultos Com Diabetes Mellitus 2 Da estratégia Saúde Da Família Collen Do Município Cambuí, Minas Gerais. Belo Horizonte. 2016.

23. ZIMMET P, Alberti GK. Epidemiology of Diabetes: Status of a Pandemic and Issues Around Metabolic Surgery. Diabetes care. 2016; 39(6):878-83. 